\title{
EVALUACIÓN DIAGNÓSTICA DE UN NOMOGRAMA DE PREDICCIÓN DE PREECLAMPSIA
}

\author{
Raúl C Alegría-Guerrero ${ }^{1,2, a}$, Carlos A. Gonzales-Medina 2,b,c,d
}

\begin{abstract}
RESUMEN
Objetivo. Determinar el estudio de pruebas diagnósticas para el nomograma de predicción propuesto en la estimación del riesgo de preeclampsia. Materiales y métodos. Estudio primario de investigación clínica observacional de casos-controles anidado y de validación del índice de predicción propuesto con el diseño de un nomograma. Se realizó en el Hospital Guillermo Almenara Irigoyen-EsSalud, Lima-Perú, Unidad de Medicina Fetal. Participaron pacientes gestantes asintomáticas de la Red Asistencial Almenara-EsSalud entre el primer o el segundo trimestre de gestación (antes de las 20 semanas) que desarrollaron o no al final del seguimiento preeclampsia severa. Se evaluó los parámetros predictores clínicos, de laboratorio y del doppler de las arterias uterinas. Los datos obtenidos se analizaron bajo un modelo de regresión logística binaria multivariante para construir la ecuación de predicción y el nomograma de predicción. Además, se realizó el análisis de curvas ROC. Resultados. El nomograma de predicción incluye parámetros clínicos, de laboratorio y doppler. Tiene una área bajo la curva (AROC) de 0,82, $p<0,001$. El nomograma propuesto califica los riesgos en alto, intermedio y bajo. Alcanza una sensibilidad de $82,2 \%$, una especificidad de $75,1 \%$, un VPN $86,6 \%$ y un VPP de $98,8 \%$. Conclusiones. El nomograma de predicción de riesgo de preeclampsia predice el desarrollo de preeclampsia con una alta sensibilidad y pude ser utilizado en la práctica clínica.
\end{abstract}

Palabras clave: Preeclampsia; Predicción;Nomograma (Fuente: DeCS BIREME).

\section{DIAGNOSTIC EVALUATION OF A PREDICTION NOMOGRAM FOR PREECLAMPSIA}

\begin{abstract}
Objective. Determine the study of the diagnostic test of the prediction nomogram for the risk of preeclampsia. Material and method: A nested case-control analytical observational study and validation of the proposed prediction index was performed with the design of a nomogram. It was performed at the Guillermo Almenara Irigoyen-EsSalud Hospital, LimaPeru, and Fetal Medicine Unit. Participated asymptomatic pregnant patients of the Almenara-EsSalud Healthcare Network between the first or second trimester of pregnancy that developed or not at the end of the follow-up severe preeclampsia. The clinical, laboratory and Doppler predictors of the uterine arteries were evaluated. The data obtained were analyzed under a multivariate binary logistic regression model to construct the prediction equation and the prediction nomogram. In addition, the ROC curve analysis was performed. The prediction nomogram includes clinical, laboratory and Doppler parameters. It has an area under the curve (AROC) of $0,82, p<0,001$. The proposed nomogram qualifies risks in high, intermediate and low. It reaches a sensitivity of $82,2 \%$, a specificity of $75,1 \%$, a VPN $86,6 \%$ and a PPV of $98,8 \%$. Conclusions: The nomogram to predictor of risk of severe preeclampsia proposed preeclampsia predicts the development with high sensitivity can be used in clinical practice.
\end{abstract}

Key words: Preeclampsia; Prediction; Nomogram (Source: MeSH NLM).

\section{INTRODUCCIÓN}

Uno de los Objetivos del Desarrollo Sostenible de la Organización Mundial de la Salud (OMS) es reducir la razón de mortalidad materna mundial a menos de 70 por 100 mil nacidos vivos entre el 2016 y 2030. Así, más de medio millón ${ }^{1,2}$ de muertes anuales en el mundo son por causas relacionadas al embarazo. En América
Latina y El Caribe, las tasas de mortalidad materna persisten elevadas ${ }^{3}$. Y en el Perú se registró en 92,7\% el año $2011^{2,4}$.

Los desórdenes hipertensivos del embarazo constituyen el $28 \%$ de las muertes maternas, ocupando el segundo lugar después de la hemorragia ${ }^{5,6} . Y$ dentro de ellos, la preeclampsia genera morbimortalidad materna y fetal

\footnotetext{
Hospital Nacional Guillermo Almenara Irigoyen (HNGAI) EsSalud, Lima - Perú.

Médico Cirujano Especialista en Ginecología y Obstetricia Facultad de Medicina San Fernando, Universidad Nacional Mayor de San Marcos (UNMSM), Miembro de la Sociedad Peruana de Ginecología y Obstetricia SPOG, Perú.

a Jefe del Servicio de Ginecología y Obstetricia de Alto Riesgo de la Unidad de Medicina Fetal HNGAI.

b Fellow Junior ACOG, American College of Obstetricians and Gynecologists, United States of America.

Unidad Funcional de Investigación del Instituto Nacional Materno Perinatal- Lima, Perú.

Maestría en Investigación Clínica, Universidad San Martin de Porres. Lima-Perú.
}

Citar como: Alegría-Guerrero RC, Gonzales-Medina CA. Evaluación diagnóstica de un nomograma de predicción de preeclampsia. Rev Peru Investig Matern Perinat 2018; 7(2):21-30.

https://doi.org/10.33421/inmp.2018114

Recibido: 10-11-18 Aprobado: 08-12-18 
importante en países subdesarrollados ${ }^{6,7}$.Asimismo, es la causa principal de restricción del crecimiento intrauterino (RCIU) $)^{7}$.

La preeclampsia es un desorden mutisistémico que es clínicamente definido actualmente con hipertensión asociada o no a proteinuria después de las 20 semanas de embarazo ${ }^{7,8}$. Además, de la presión arterial media (PAM) elevada, el Índice de Masa Corporal (IMC) aumentado y la perdida de proteínas en orina de 24 horas son buenos predictores en el primer trimestre de gestación, el índice de pulsatilidad promedio (IP p) de las arterias uterinas en el doppler materno y los marcadores bioquímicos aumentan la sensibilidad para la predicción de preeclampsia ${ }^{8}$. Generalmente, los niveles de factores proangiogénicos están disminuidos mientras que los factores antiangiogénicos están incrementados en la circulación materna antes del inicio de la enfermedad ${ }^{6,7}$. Por ello, diversos marcadores propuestos como la proteína plasmática asociada a la gestación (PAPP-A) y los niveles de sFlt-1 aumentados ${ }^{7}$ han demostrado que combinados son buenos predictores de la enfermedad ${ }^{8,9}$, pero el acceso a estas pruebas es aun costoso. Por tanto, sería necesario desarrollar un modelo predictivo en el que considere la historia clínica obstétrica, análisis de laboratorio y el doppler promedio de las arterias uterinas en gestantes asintomáticas antes del inicio de la enfermedad. En el 2014 se desarrolló un índice de predicción de preeclampsia ${ }^{10}$ para la pacientes que ingresaban al Departamento de Ginecología y Obstetricia y la Unidad de Medicina Fetal con Diagnóstico Prenatal del Hospital Nacional Guillermo Almenara Irigoyen (HNGAI) EsSalud. Este índice posteriormente se reevaluó y se rediseño en un estudio posterior mediante la elaboración de un nomograma de predicción propuesto. Sin embargo, dada su potencial capacidad predictiva y su aceptación por los médicos de nuestro servicio como una herramienta útil se decidió someter a prueba dicho instrumento y someterlo a validación como herramienta diagnóstica y predictiva. Por ello, el objetivo del estudio fue desarrollar el estudio de pruebas diagnósticas de un nomograma de predicción propuesto para el riesgo de preeclampsia.

\section{MATERIALES Y METODOS}

Diseño del estudio. Inicialmente se realizó un estudio caso-control anidado ${ }^{11}$ en el que se obtuvo una medición basal de los parámetros clínicos, de laboratorio y del doppler de las arterias uterinas. Luego, se seleccionó a las pacientes en función al efecto (desarrollo de la enfermedad). De tal manera que los casos eran las pacientes que presentaban preeclampsia y los controles los que no de manera anidada. Luego, se realizó el proceso de validación que incluyó la elaboración de la ecuación de predicción con el nomograma propuesto y finalmente se realizó el estudio de pruebas diagnósticas del mismo. Población y muestra. La población incluyo gestantes asintomáticas atendidas en el periodo de un año en Departamento de Ginecología y Obstetricia y la Unidad de Medicina Fetal y Diagnóstico Prenatal del Hospital Nacional Guillermo Almenara Irigoyen (HNGAI) EsSalud, Lima-Perú. El tamaño muestral se realizó según el muestreo probabilístico para estudios de casos y controles que utilizan regresión logística. Se determinó la potencia estadística en $80 \%$ cuando se incluye tres controles por cada caso. El muestreo se estableció con un nivel de confianza del $95 \%$ y un margen de error de $\pm 1.5 \%$. Se consideró una tasa de pérdida y reposiciones de $5 \%$. La unidad análisis fue cada gestante asintomática que cumplía los criterios de inclusión y exclusión del protocolo del estudio según los nuevos criterios diagnósticos ACOG 2013 y los actualizados para la publicación de enero del 2019 ${ }^{12,35}$. Tamaño de la muestra. El tamaño muestral se determinó con el programa estadístico de la calculadora GRANMO disponible en internet bit.ly/calculadoraGranmo. Se determinó según la literatura biomédica la proporción de controles expuestos al factor con un valor de $25 \%$, con una razón de casos por controles de 3 a 1 y además se consideró una proporción prevista de pérdidas del seguimiento del $5 \%$ calculándose 119 casos y 357 controles. Procedimientos. Existo 5 fases para el desarrollo del estudio: 1ra fase: Captación de pacientes, firma de consentimiento informado y consejería. 2da fase: Recolección y toma de muestras. 3ra fase: Procesamiento de muestras y toma de las ecografías doppler. 4ta fase: Monitorización y seguimiento de pacientes: En esta fase se determinó según la historia clínica el diagnostico final de las pacientes luego del seguimiento así como su monitorización según las Guías de práctica clínica del Servicio de Obstetricia de Alto Riesgo. Asimismo, se consideró el plan de análisis y desarrollo del estudio de pruebas diagnósticas del nomograma propuesto. 5ta fase: Manejo y Consejería de las pacientes con resultado de pre eclampsia o embarazo normal al término de la gestación: Las dos últimas fases se realizaron en los consultorios externos y/o emergencia obstétrica del departamento del Servicio de Ginecología y Obstetricia del Hospital. Plan de Análisis estadístico. El análisis estadístico se realizó en dos fases. La primera comprendió la descriptiva y exploratoria en la que se analizó la frecuencia de los datos categóricos, según las características de presencia o no de la enfermedad. $Y$ se analizó las variables cualitativas según prevalencias y proporciones. La medida de fuerza de asociación fue el Odds Ratio (OR) y Chi-cuadrado $\left(x^{2}\right)$. En las variables numéricas se calculó el promedio, desviación estándar y el rango del intervalo cuartilar (RIQ). La segunda fase comprendió la analítica en la que se exploró la distribución de los datos para determinar la normalidad. Para ello, se usó métodos gráficos (grafico Q-Q plot y Box-plot) y la prueba de Kolmogorov Smirnov. Luego, 
se realizó el estudios de pruebas diagnósticas y se obtuvo la sensibilidad, especificidad, VPP, VPN, LR+/likelihood ratios positivo y negativo para la evaluación de la ecuación de predicción. Así mismo, con el análisis de curvas ROC se determinó los puntos de corte óptimos y las áreas bajo la curva respectivas. En todos los casos se estimó el intervalo de confianza al $95 \%$ de cada uno de los parámetros. El desarrollo de la presencia o ausencia de preeclampsia (variable dependiente dicotómica) en el seguimiento se determinó usando un modelo multivariado de regresión logística binaria ${ }^{13}$.Se aplicó el test de Wald-Hosmer y los OR ajustados según el modelo usando el método enter. Además, se usó el método Kaplan Meir en función de las probabilidades acumuladas en relación con el tiempo. Y se determinó la comparación de las curvas con la Regresión de Cox calculando el Hazard Ratio con su respectivo intervalo de confianza. Todo el análisis estadístico se realizó en el paquete estadístico IBM-SPSS versión 17.0 Para la construcción y diseño del normograma de predicción se diseñó en el paquete $\mathrm{R}$ package with the Survival, Design,Hmisc, Party, and Lexis libraries (http://lib.stat. cmu.edu/R/CRAN/). Y se realizaron precisiones graficas en CoreIDRAW Graphics Suite X8. El nivel de significancia estadística para todas las pruebas estadísticas fue un $p<0.05$. Ética. Las participantes fueron invitadas a participar en el estudio de forma voluntaria y se aplicó un consentimiento informado; cumpliendo en todo momento del estudio con los principios de autonomía, justicia y beneficencia. Los datos requeridos para el estudio fueron codificados y almacenados en un archivo electrónico con acceso restringido, para proteger la confidencialidad de los participantes. De igual forma, el estudio se realizó siguiendo el código de Ética del Colegio Médico del Perú, tomando en cuenta el Artículo $N^{\circ} 51$, dentro del cual se establece que el médico debe respetar y hacer respetar el derecho del paciente a la confiabilidad de sus datos médicos, personales que le conciernan y que la discusión del caso, las consultas, las exploraciones y el tratamiento sean confidenciales y conducidos con la discreción que se merecen. Así mismo, el protocolo de investigación fue evaluado por un comité de ética institucional.

\section{RESULTADOS}

Se registró 119 pacientes (casos) de preeclampsia severa y 357 pacientes (controles sanos). La edad materna oscilaba entre 14 y 45 años, con un promedio $30,6 \pm 6,7$ años. Se evaluó las características clínicas, de laboratorio y del doppler de las arterias uterinas en los diferentes grupos. La nuliparidad y el antecedente de preeclampsia tienen un OR 4,51 IC95\% $(2,34-6,91)$ y OR 6,41 IC95\%(3,41-8,31) respectivamente para desarrollar preeclampsia. La edad materna mayor de 40 años y menor de igual a 15 años tuvo similares
OR 7,21 IC95\%(3,45-9,34) OR 2,28 IC $95 \%(2,49$ $5,48)$ respectivamente existiendo diferencia estadísticamente significativa. No ocurrió lo mismo con los parámetros medidos al inicio del seguimiento como glucosa, urea, creatinina, hemograma, estudio de coagulación, albumina, enzimas hepáticas y recuento plaquetas en los que no hubo diferencias estadísticamente significativas en ambos grupos. Sin embargo, existió una ligera disminución de plaquetas no significativa en el grupo de casos en comparación con los controles.

Se halló que existe diferencia estadísticamente significativa entre el promedio del IP de las arterias uterinas en el primer trimestre en la ecografía de 11 a $13+6$ semanas siendo mayor en el grupo de casos (Tabla 4). EI IP promedio mayor del percentil 90 y 95, se asoció a un mayor riesgo de preeclampsia severa OR 6,76 (IC 95\% 4,08 a 9,18) en comparación con los controles. La PAM promedio en el primer y segundo trimestre (antes de las 20 semanas) mayor igual a 106 mmHg tuvo un OR: 3,48 (IC 95\% 2,57 a 4,69). El valor promedio de la proteína en orina de 24 horas durante el primer trimestre, se asoció con un OR 3,57 (IC 95\% 2,15 a 4,56). La presencia de otras comorbilidades $u$ condiciones obstétricas actuales en el grupo de casos aumenta el riesgo de desarrollar preeclampsia OR 4,58 (IC 95\% 2,17 a 4,59). Construcción de la ecuación de predicción y el nomograma. La población de estudio se distribuyó en dos grupos uno denominado diseño y otro llamado validación.Y se distribuyó por el método de polinomios factoriales. La construcción de la ecuación de predicción se realizó mediante la regresión logística binaria multivariada. Con la regresión logística se expresó la probabilidad de que ocurra el evento en cuestión (desarrollo de preeclampsia) en función de ciertas variables que son relevantes y han demostrado que ofrecen individualmente altos niveles de predicción. El modelo inicial que se seleccionó incluyó 8 variables, de tal manera que se pudo obtener una fórmula para estimar el riesgo. Esta ecuación tiene un área bajo la curva ROC de $0.82 \pm 0.02$ (IC 95\%=0.85; 0.92) (ver Figura 2). Esta función exponencial es una expresión simplificada que corresponde en elevar el número neperiano (e) a la potencia contenida dentro del paréntesis, siendo (e) el número o constante de Euler, o base de los logaritmos neperianos (cuyo valor aproximado a la milésima es 2,718$)$. La ecuación de predicción se expresa a continuación:

\footnotetext{
Logit $(p)=156.3+10 x I M C \geq 25+36 x P A M \geq 90$

$+1.5 x A n t P E+4,5 x E d a d+14 x I P$ AtU $\geq 95+$

19xProteinuria $\geq 300+2.3 \times$ Nuliparidad $+0,23 x$ Otras

Comorbilidades
} 
NOMOGRAMA DE PREDICCIÓN DE PREECLAMPSIA

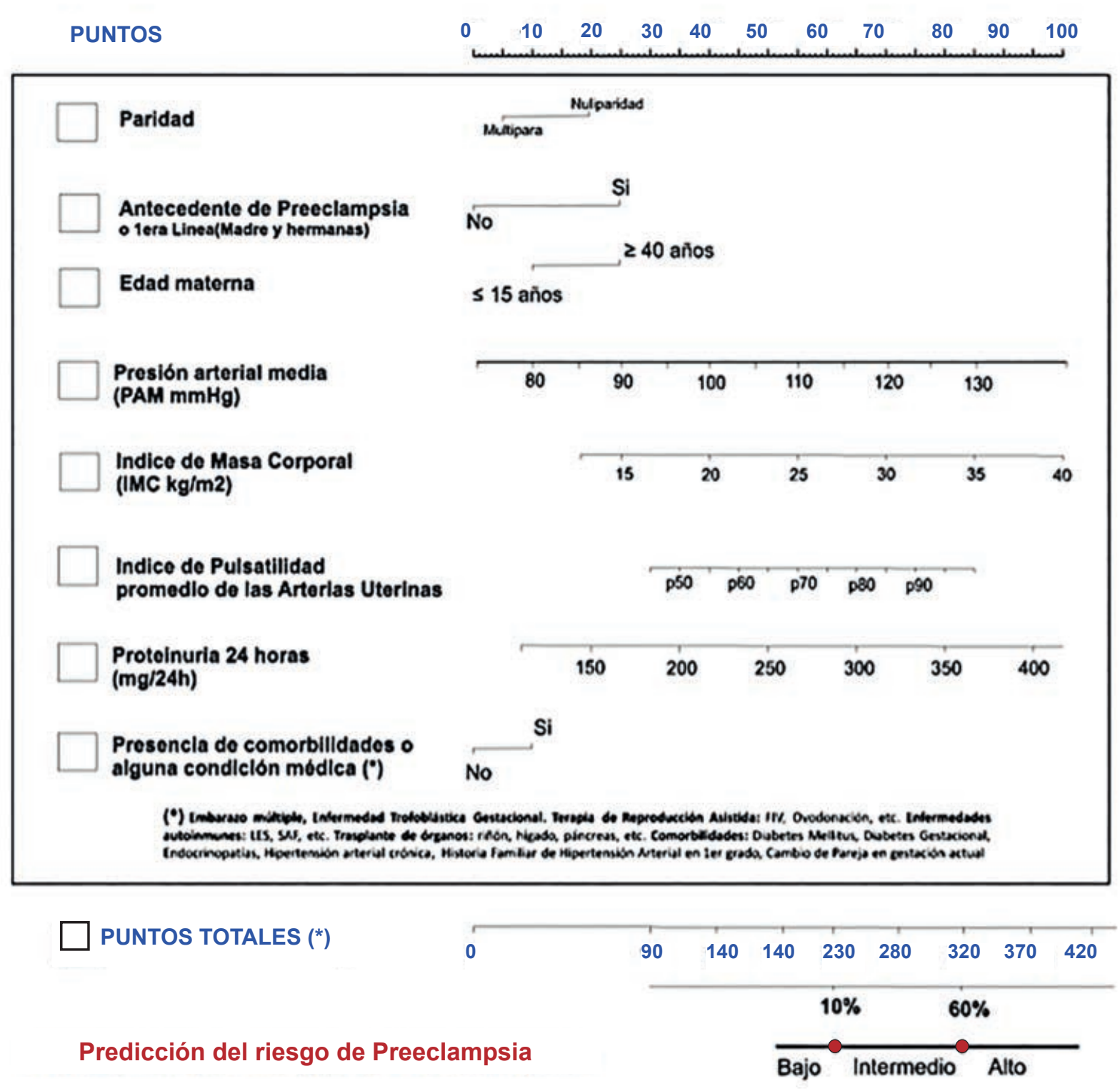

Figura 1. Nomograma de predicción de preeclampsia, se muestra las variables involucradas y el porcentaje de predicción de riesgo de preeclampsia según los puntajes totales.

La construcción de la ecuación de predicción se basó en el modelo de regresión logística y se diseñó con los valores de los Odds ratios (OR) de cada factor de predicción de modo que estos $\mathrm{OR}$ recibieron un peso proporcional a su logaritmo, y así se determinó el puntaje promedio de cada parámetro. Además, se evaluó la linealidad del Logit, por lo que se recurrió a los métodos gráficos de los diagramas de dispersión propios del método estadístico.

El área bajo la curva ROC fue 0.82 en el grupo validación y también, luego de hacer el proceso de validación cruzada con los diferentes subconjuntos y evaluándose la precisión diagnostica, del sistema de puntación del Nomograma de predicción para aquellos pacientes que desarrollaron o no la enfermedad. Además, para la validación del nomograma se realizó la concordancia por el método de remuestreo bootstrap que estimó el intervalo de confianza. Para la calibración del modelo se uso ó $=0$ y $\beta=1$.

En la ecuación de predicción de riesgo de preeclampsia severa se incluye parámetros de ecodoppler, de laboratorio y clínicos. Finalmente, se ordenan los puntajes en una la escala numérica (Ver Figura 1) colocando al valor de 250 puntos como punto de corte discrimínate del índice propuesto según el valor obtenido en la curva ROC. (Ver Figura N2).Punto de corte en el que se obtiene la mejor sensibilidad y especificidad de la prueba.

Con el valor de corte de 250 puntos el nomograma de predicción de preeclampsia alcanza una sensibilidad 
Tabla 1. Análisis de los factores de riesgo relacionados con preeclampsia severa en el modelo final de regresión logística binaria multivariada mostrando puntos de corte.

\begin{tabular}{lccc}
\hline Variables & $\begin{array}{c}\text { OR } \\
\text { ajustado* }\end{array}$ & IC 95\% \\
\hline IP arteria uterinas promedio & & & \\
< p 95 & 1 & & \\
$\geq$ p 95 & 6.757 & 4,081 & 9,180
\end{tabular}

Presión arterial media

$\begin{array}{lccc}<90 \mathrm{mmHg} & 1 & & \\ \geq 90 \mathrm{mmHg} & 3.476 & 2.576 & 4.690 \\ \text { Proteína en orina de 24 h } & & & \\ <300 \mathrm{mg} & 1 & & \\ \geq 300 \mathrm{mg} & 3,57 & 2,151 & 4,510\end{array}$

Índice de Masa Corporal

$\begin{array}{lccc}<25 \mathrm{~kg} / \mathrm{m} 2 & 1 & & \\ \geq 25 \mathrm{~kg} / \mathrm{m} 2 & 4.081 & 2.772 & 6.008 \\ \text { Antecedente de preeclampsia } & & & \\ \text { No } & 1 & & \\ \text { SI } & 5.234 & 2.968 & 9.233\end{array}$

Presencia de comorbilidades o condición médica actual

$\begin{array}{lccc}\text { NO } & 1 & & \\ \text { SI } & 0.750 & 0.156 & 3.601 \\ \text { Edad materna }(\geq 40 a \quad y \leq 15 a) & & & \\ \text { NO } & 1 & & \\ \text { SI } & 0.008 & 0.200 & 1.088\end{array}$

\section{Paridad( Nulípara)}

\begin{tabular}{lccc} 
NO & 1 & & \\
SI & 1,031 & 0.300 & 4.088 \\
\hline
\end{tabular}

OR*: odds ratio con análisis bivariado y multivariado

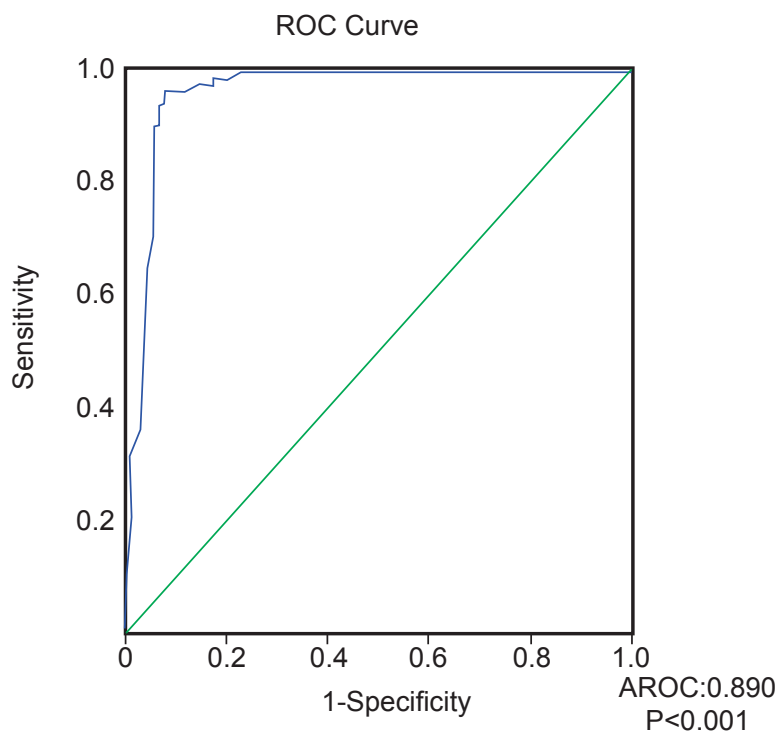

Figura 2. Curva ROC de la ecuación de predicción de riesgo de preeclampsia severa que incluye los parámetros seleccionados en función logit obtenida. Valor de AROC de $0.82 \pm 0.02$ (IC $95 \%=0.85 ; 0.92$ )

del $82,2 \%$, una especificidad de $75.1 \%$, un VPN $86.6 \%$, de VPP de $78.8 \%$, un LR +19.5 y un LR- de 0.04 .Y el valor del AROC del índice de predicción es 0.82 . Se obtuvo la ecuación de predicción mediante la fórmula en función Logit y se consideró a 8 variables de ella para la elaboración del nomograma de predicción de riesgo.

Se determinó que durante el tiempo de aparición del evento de interés (preeclampsia severa) cada una de las categorías de clasificación (nivel alto, intermedio o bajo) acumula progresivamente en el tiempo una probabilidad de que aparezca el evento de interés. Además, se determina la probabilidad que tiene cada categoría de clasificación. (Ver figura $N^{\circ} 1$ ). Según esta valoración

Tabla 2. Área bajo la curva (AROC) de las variables analizadas en el nomograma para predecir preeclampsia severa.

\begin{tabular}{|c|c|c|c|c|c|}
\hline \multirow[b]{2}{*}{ Variable de estudio } & \multirow[b]{2}{*}{ Área } & \multirow[b]{2}{*}{$\begin{array}{l}\text { Error } \\
\text { tipo a }\end{array}$} & \multirow[b]{2}{*}{ Sig. asintótica } & \multicolumn{2}{|c|}{ IC $95 \%$} \\
\hline & & & & Límite inferior & $\begin{array}{l}\text { Límite } \\
\text { superior }\end{array}$ \\
\hline IP u promedio $\geq$ p 95 & 0.951 & 0.014 & 0.001 & 0.923 & 0.979 \\
\hline $\mathrm{PAM} \geq 90 \mathrm{mmHg}$ & 0.902 & 0.019 & 0.002 & 0.864 & 0.939 \\
\hline Proteinuria $24 \mathrm{~h} \geq 300 \mathrm{mg}$ & 0.968 & 0.009 & 0.001 & 0.950 & 0.987 \\
\hline Paridad & 0.497 & 0.036 & 0.940 & 0.427 & 0.568 \\
\hline $\mathrm{IMC} \geq 25 \mathrm{~kg} / \mathrm{m}^{2}$ & 0.877 & 0.029 & 0.001 & 0.819 & 0.934 \\
\hline Edad materna ( $\geq 40 a \quad y \leq 15 a)$ & 0.665 & 0.033 & 0.001 & 0.601 & 0.730 \\
\hline Antecedente de Preeclampsia & 0.492 & 0.026 & 0.930 & 0.427 & 0.587 \\
\hline Presencia de comorbilidades o condición médica actual & 0.397 & 0036 & 0.940 & 0.307 & 0.568 \\
\hline Nomograma de predicción & $0.820^{*}$ & 0.031 & 0.001 & 0.857 & 0.928 \\
\hline
\end{tabular}

IP u: Índice de Pulsatilidad de las arteria uterinas; PAM: Presión arterial media; IMC: Índice de Masa Corporal, ${ }^{\star} \mathrm{p}<0.001$ 
Tabla 3. Valores diagnósticos de los parámetros considerados en el nomograma de predicción de preeclampsia propuesto.

\begin{tabular}{|c|c|c|c|c|c|c|c|c|c|c|c|}
\hline Parámetros & s & E & VPP & VPP & PFP & PFN & LR + & IC $95 \%$ & LR - & \multicolumn{2}{|c|}{ IC $95 \%$} \\
\hline $\begin{array}{l}\text { IP Arterias uterinas } \\
\text { promedio } \geq 95\end{array}$ & $92,8 \%$ & $95,4 \%$ & $86,8 \%$ & $96,9 \%$ & $4,6 \%$ & $9,2 \%$ & 19,90 & 11,40 a 34,73 & 0,10 & 0,05 & a 0,19 \\
\hline PAM $\geq 90 \mathrm{mmHg}$ & $92,6 \%$ & $91,4 \%$ & $77,7 \%$ & $97,2 \%$ & $8,6 \%$ & $8,0 \%$ & 10,63 & a 15,79 & 0,09 & 0,04 & a 0,18 \\
\hline Proteinuria $24 \mathrm{~h} \geq 300 \mathrm{mg}$ & $78,8 \%$ & $66,6 \%$ & $88,3 \%$ & $93,0 \%$ & $3,4 \%$ & $21,8 \%$ & 22,84 & 11,90 a 43,82 & 0,23 & 0,15 & a 0,34 \\
\hline $\mathrm{IMC} \geq 25 \mathrm{~kg} / \mathrm{m}^{2}$ & $88,2 \%$ & $90,4 \%$ & $77,3 \%$ & $92,7 \%$ & $7,6 \%$ & $21,8 \%$ & 10,28 & 6,65 a 15,89 & 0,24 & 0,16 & a 0,35 \\
\hline Antecedente de preeclampsia & $69,9 \%$ & $76,2 \%$ & $82,8 \%$ & $86,6 \%$ & $3,8 \%$ & $44,8 \%$ & 14,51 & 7,68 a 27,43 & 0,47 & 0,37 & a 0,59 \\
\hline Edad materna ( $\geq 40 a \quad y \leq 15 a)$ & $79,2 \%$ & $86,6 \%$ & $88,3 \%$ & $93,0 \%$ & $3,4 \%$ & $21,8 \%$ & 22,84 & 11,90 a 43,82 & 0,23 & 0,15 & a 0,34 \\
\hline Paridad & $78,2 \%$ & $86,2 \%$ & $82,8 \%$ & $86,6 \%$ & $3,8 \%$ & $44,8 \%$ & 14,51 & 7,68 a 27,43 & 0,47 & 0,37 & a 0,59 \\
\hline $\begin{array}{l}\text { Presencia de comorbilidades o } \\
\text { condición médica actual }\end{array}$ & $78,2 \%$ & $72,4 \%$ & $77,3 \%$ & $92,7 \%$ & $7,6 \%$ & $21,8 \%$ & 10,28 & a 15,89 & 0,24 & 0,16 & a 0,35 \\
\hline Nomograma de predicción & $82,2 \%$ & $75,1 \%$ & $86,6 \%$ & $78,8 \%$ & $4,9 \%$ & $3,4 \%$ & 19,53 & 11,48 a 33,23 & 0,04 & 0,01 & a 0,11 \\
\hline
\end{tabular}

* S: Sensibilidad, E: Especificidad, VPP: Valor predictivo positivo, VPN: Valor predictivo negativo, PFP: Proporción de falsos positivos, PFN, proporción de falsos negativos, LR +: likelihood ratios positivo, LR- likelihood ratios negativo. IC 95\%: intervalo de confianza al $95 \%$.

Tabla 4. Valores promedios de los índices de resistencia y pulsatilidad en el estudio doppler de las arterias uterinas medidas en el segundo trimestre de gestación.

\begin{tabular}{lccc}
\hline \multicolumn{1}{c}{ VARIABLES } & $\begin{array}{c}\text { Casos } \\
\mathrm{x} \pm \mathrm{DS}\end{array}$ & $\begin{array}{c}\text { Controles } \\
\mathrm{x} \pm \mathrm{DS}\end{array}$ & $\mathbf{p}^{*}$ \\
\hline $\begin{array}{l}\mathrm{IR} \text { de las arterias } \\
\text { uterinas }\end{array}$ & $0.73 \pm 0.06$ & $0.72 \pm 0.05$ & 0.808 \\
$\begin{array}{l}\text { IP promedio de las } \\
\text { arterias uterinas }\end{array}$ & $1.84 \pm 0.39$ & $1.05 \pm 0.29$ & $<0,01$ \\
\hline
\end{tabular}

IR: Índice de resistencia, IP: índice de pulsatilidad

$\mathrm{x} \pm$ DS: media (desviación estándar).

* $=\mathrm{t}$ de student

si las pacientes obtiene una puntuación de "alto riesgo" tiene una probabilidad de predicción de riesgo estimada superior al $60 \%$, si tienen obtienen una puntación de "intermedio riesgo" es $10 \%$ al $59 \%$ y si es "bajo riesgo" de $9 \%$ a menos.

La evaluación de las pruebas diagnósticas con sus diferentes indicadores de predicción se realizó de forma individual y en forma global según el nomograma propuesto considerando como gold standar el diagnostico final de preeclampsia severa (Ver tabla 2 y 3 ).

\section{DISCUSIÓN}

En el presente estudio se confirma que los parámetros asociados de manera conjunta en el nomograma de predicción propuesto han demostrado ser buenos predictores del diagnóstico final de preeclampsia. Y tienen una alta sensibilidad y una tasa de detección buena para ser usados en la práctica clínica.
Los antecedentes familiares y personales de diabetes mellitus tipo 2, hipertensión arterial y asma bronquial no se asociaron estadísticamente con el riesgo de preeclampsia. Se reporta en otros estudios que el antecedente de padres con historia de diabetes tiene un OR 3,4 (IC 95\% $1,4$ a 8,4$)^{14}$. La diabetes por tener una base inmunológica podría activar o desactivar un conjunto de poligenes que se hallan en el ADN de la madre con repercusiones desde un punto de vista epigenético en el fenotipo final.

El antecedente de cesárea se asoció con el riesgo de preeclampsia pero la asociación no fue estadísticamente significativa. Sin embargo, un estudio realizado en México ${ }^{32}$ determinó una asociación muy alta con cifras del OR en 16.20 IC 95\% $(4,5-131,3)$. Este dato es interesante y controversial que amerita una mejor revisión, porque se cree que el escobillanaje trans cesárea estaría implicado en el daño de la decidua basal y del sistema de arterias espirales en una siguiente gestación. La preeclampsia afecta a menudo a mujeres jóvenes y nulíparas mientras que las pacientes mayores tienen mayor riesgo de desarrollar hipertensión crónica con preeclampsia agregada ${ }^{14,15}$. Un estudio halló ${ }^{15}$ que la incidencia de pacientes era mayor en el grupo de 15 a 20 años y en el de 45 a 50 años. Asimismo, en aquellas pacientes en las que coexistían condiciones como obesidad, hipertensión, historia familiar de enfermedades cardiacas, sedentarismo, diabetes mellitus (en especial en pacientes de raza negra), los casos aumentaban con la presencia de más comorbilidades. La edad materna avanzada es otro factor de riesgo independiente para preeclampsia (edad materna $\geq 40$ : OR: 1.96; IC 95\%: 1.34-2.87) $)^{16,17}$. Esto puede deberse a que las mujeres de edad avanzada 
tienen factores de riesgo adicionales, como diabetes mellitus (OR: 3.56; IC 95\%: 2.54-4.99) e hipertensión crónica que hacen que los riesgos relativos se sobrestimen más ${ }^{17}$.

El periodo intergenésico de más de 10 años genera tres veces más riesgo de tener preeclampsia ${ }^{17}$.Cifras similares se documentan en un estudio de casos y controles donde se encontró que el riesgo de presentar preeclampsia aumentó cuatro veces más en las mujeres que tuvieron periodo intergenésico corto $(\mathrm{OR}=4.62$, IC $95 \%=1.2-18.5)^{17}$.

El índice de masa corporal (IMC) constituye uno de los factores de riesgo relacionados con preeclampsia de tal forma que la relación entre el peso de la madre y el riesgo de preeclampsia es progresiva ${ }^{18}$. Por ello el riesgo de un IMC mayor a $35 \mathrm{~kg} / \mathrm{m} 2$ pre gestacional y durante el primer trimestre incrementa 13 veces el riesgo de enfermar ${ }^{18}$. Así, en México y Cuba ${ }^{19}$ la obesidad pre gestacional (IMC $\geq 27 \mathrm{~kg} / \mathrm{m} 2$ ) es un factor que aumenta cuatro veces el riesgo de preeclampsia. La obesidad conlleva a un síndrome metabólico que contribuiría a la resistencia a la insulina y no permitiría la activación de las cascadas de degradación catabólica. Asimismo, se sabe que la insulina actúa sobre las células periféricas para estimular la captación utilización y almacenamiento de glucosa y por otro lado desde una perspectiva corporal, la insulina tiene un efecto en la disminución de las grasas.

El valor de la Presión Arterial Media (PAM) en el 1er trimestre fue mayor en el grupo de pacientes con preeclampsia que en el grupo control no encontrándose diferencias estadísticamente significativas. Pero, el valor promedio de la PAM en el 2er trimestre (antes de las 20 semanas) fue mayor en el grupo de pacientes con preeclampsia que en el grupo control encontrándose diferencias estadísticamente significativas. Aunque una elevación de la presión arterial diastólica o PAM en el segundo trimestre puede predecir adecuadamente la aparición del síndrome hipertensivo del embarazo, este cambio no está asociado a una mayor morbimortalidad perinatal $^{20}$.

Un estudio multicentrico ${ }^{20}$ demuestra que la PAM en el primer o segundo trimestre superior a $90 \mathrm{mmHg}$ se asocia con mayor riesgo de preeclampsia. La PAM en el primer o segundo trimestre de gestación, según una revisión sistemática publicada en el 2008, es una de las mejores variables predictoras de preeclampsia ${ }^{21}$.

Por otra parte la PAM medida en el segundo trimestre de gestación tiene una sensibilidad de $92.0 \%$ y una especificidad de $91.4 \%$ con un (LR+:7.16, LR-: 0.09).Así, valores similares se hallaron en un metaanalisis ${ }^{21}$ donde la PAM mayor o igual a $90 \mathrm{mmHg}$ en el segundo trimestre demostró tener una sensibilidad de $62 \%$ y una especificidad de $82 \%$ (LR+:3.5, LR-: 0.5) mientras para PAM mayor o igual de $85 \mathrm{mmHg}$ demostró tener una la sensibilidad y especificidad de 52 y $84 \%$ respectivamente. Finalmente, en poblaciones de alto riesgo de preeclampsia una presión arterial diastólica $\geq 75 \mathrm{mmHg}$ registrada entre las 13 a 20 semanas gestacionales mostró un LR+ de 2.8 y un LRde 0.4 de desarrollar la enfermedad posteriormente. La conclusión es que la medida de la presión arterial en el primer y segundo trimestre tiene una capacidad predictiva muy importante para preeclampsia ${ }^{20,21}$. Por ello debe existir una rigurosidad en la precisión de la toma de la presión arterial.

Los valores de los Índice de Resistencia (IR) y del Índice de Pulsatilidad promedio (IP p) de las arterias uterinas en el estudio doppler, medidos en el segundo trimestre fueron en promedio superior en los casos de preeclampsia que en los controles. Además, en el análisis de los factores de riesgo relacionados con preeclampsia el IP promedio de las arterias uterinas mayor igual al percentil 95 (y desde el percentil 90) para la edad gestacional, se asoció a un mayor riesgo de desarrollar preeclampsia severa en comparación con los controles. Así en una revisión sistemática publicada el $2008^{21}$ en la que se incluyó 74 estudios, el Doppler de las arterias uterinas fue evaluado entre las semanas 18 a 24 de gestación y los valores del IR medidos en el segundo trimestre fueron superiores al valor estimado del percentil 90. Así, se obtuvo una sensibilidad de $83 \%$ y una especificidad de $72 \%$ (LR+:3.0, LR-: 0.24). Además, el valor del índice de pulsatilidad para predecir preeclampsia severa tiene una sensibilidad de $78 \%$ y especificidad de $95 \%$ cuando se evaluaba en el segundo trimestre de gestación.

Los valores IP promedio de las arterias uterinas superiores al percentil 95 para predecir el desarrollo de preeclampsia muestran una sensibilidad de $90,8 \%$ y una especificidad de $95,4 \%$.Asimiso ,un VPP de $86,8 \%$, un VPN de $96,9 \%$ y un LR+ de 19.9 (IC95\% 11.4 a 34.7) siendo muy similar a los cálculos de los valores de predicción de un estudio multicentrico en el que las pacientes involucradas eran categorizadas con un IP mayor al percentil 95 obteniendo una sensibilidad de $77.4 \%$, una especificidad de $95.2 \%$, un VPP de $6.0 \%$, un VPN de $99.9 \%$ y un LR+ de 16.1(IC95\% 12.3-19.1). Asimismo, se determinó que el IP promedio mayor de 1.6 obtenido alrededor de la semana 23 , se asociaba a una mayor tasa de embarazos que desarrollaban preeclampsia ${ }^{22}$.

En un estudio peruano concluye que el IP $>2.35$, entre las 11 a 14 semanas $^{23}$ predice la preeclampsia con una sensibilidad de $66.7 \%$ y una especificidad de $95 \%$. Otra investigación determinó que los valores mayores del percentil 75 tienen un $66.7 \%$ de sensibilidad y un $75 \%$ de especificidad para la predicción. EI IP promedio 
de las pacientes fue de1.64 \pm 0.3 .2 , este hallazgo se correlaciona con los valores encontrados en diversos estudios con valores de $1.83 \pm 0.53$ a las 12 semanas y $1.71 \pm 0.47$ a las 13 semanas, valores mayores del percentili ${ }^{23,24}$. Otra investigación peruana en el $2011^{25}$ encontró que el valor del IP promedio mayor p95 entre las 11 y 14 semanas fue 2.66. Además, la capacidad predictiva del IP anormal (IP 2.66) se estableció un VPP de $33.3 \%$ (IC95\%: 0.0\% a $79.4 \%$ ), VPN de $93.0 \%$ (IC95\%: $87.9 \%$ a $98.1 \%)^{25}$.

El dato del antecedente de preeclampsia según el enfoque del estudio, se asoció significativamente a un mayor riesgo de preeclampsia severa. Los reportes existentes han asociado el antecedente de preeclampsia con un embarazo previo a un mayor riesgo de recurrencia de la enfermedad (OR: 2.91; IC 95\%:1.8$6.61)^{26}$ El riesgo de recurrencia varía dependiendo de la gravedad del cuadro de preeclampsia previo, presentado antes del embarazo índice, reportándose de 25 a $65 \%$ en pacientes con preeclampsia severa previa y de $5-7 \%$ en pacientes con preeclampsia leve comparado con la frecuencia de menos de $1 \%$ en pacientes con un embarazo previo normal ${ }^{27}$. El antecedente de preeclampsia en embarazo previo se asoció 24 veces más con el riesgo de desarrollar nuevamente dicha patología (OR $=24.80$, IC $95 \%$ : 2.8-121.8) en comparación con las pacientes que no tuvieron este antecedente ${ }^{11}$. Está bien establecido que la preeclampsia tiene tendencia familiar aunque algunos autores describen que el antecedente podría ser un factor protector por el hecho inmunológico de la "adaptación materna"12. Las gestantes nulíparas tienen $5 \%$ de riesgo de preeclampsia, pero su riesgo aumenta 5 veces si su madre tuvo preeclampsia severa. Su riesgo se reduce a $1 \%$ en el segundo embarazo del mismo esposo, si tuvo embarazo normal. El riesgo de preeclampsia es conferido de Novo en el embarazo con un nuevo esposo ${ }^{12,26,27}$.

El valor de proteínas en orina de 24 horas para el estudio de predicción ha sido evaluada recientemente. Un estudio ${ }^{28,29}$ publicado el 2010 sobre el uso de la proteína de 24 horas para predecir preeclampsia entre la semana 20 a 28 de gestación determinó que el grupo de pacientes preeclámpticas tenían un promedio $351.14 \pm 41.58 \mathrm{mg} / 24 \mathrm{~h}$ en comparación con $296.33 \pm 30.3$ $\mathrm{mg} / 24 \mathrm{~h}$ en el grupo control existiendo diferencias estadísticamente significativas entre ambos grupos. Además, un AROC de 0.846 con un valor de sensibilidad del $86 \%$ y especificidad de $72 \%$ para el punto de corte de $310 \mathrm{mg} / 24$ horas y su VPP de $26 \%$ y el VPN $98 \%$. La búsqueda actual de pruebas diagnósticas que ayuden a predecir la preeclampsia con buenos valores de predicción es fundamental para un mejor seguimiento de las pacientes con un potencial riesgo de desarrollar esta entidad.
En el estudio se confirma que los parámetros asociados de manera conjunta e integral en el normograma de predicción propuesto han demostrado ser buenos predictores del diagnóstico final de preeclampsia. $Y$ tienen una alta sensibilidad y una tasa de detección buena para utilizarse en la práctica clínica. Además, según la propuesta de invertir la pirámide de la atención prenatal clásica ${ }^{30}$ para orientar un control especializado que comience desde las 12 semanas hasta las 34 semanas en aquellas pacientes que puedan ser clasificadas como de alto o potencial riesgo de desarrollar la enfermedad y en general otras complicaciones maternas perinatales es necesario métodos de predicción como el normograma y se podrían realizar estrategias de disminución del riesgo como el estudio ASPRE.

Para el Equipo de Trabajo (ACOG -Task Force) de Hipertensión en el embarazo, los probables predictores de preeclampsia -factores demográficos, análisis bioquímicos y/o hallazgos biofísicos- tienen poca sensibilidad y especificidad. Por ello, su recomendación es realizar una historia clínica apropiada12. Lo cual apoya la idea de la mayoría de los parámetros del normograma que resultan ser son clínicos y obtenidos de la historia obstétrica.

Los valores de sensibilidad y especificidad del índice son buenos. Sin embargo, podrían potenciarse con los nuevos marcadores inmunológicos y bioquímicos para el estudio de predicción de preeclampsia que ya han sido demostrados en estudios experimentales y algunos estudios prospectivos. Por ejemplo, hay nuevos marcadores recientes como la elastografia placentaria, el ratio sFIt-1/PIGF y la IL-6. Esta última involucrada en la patogénesis de la preeclampsia sobre todo como mediadora indirecta del mecanismo de disfunción endotelial en embarazos tardíos. La actividad elevada de esta citoquina ayudaría a predecir los casos de preeclampsia severa tardía ${ }^{29,30}$. Así mismo un estudio reciente muestra el uso potencial de la tecnología del Doppler 3D para estimar un índice de vascularización placentaria como potencial predictor de preeclampsia ${ }^{32,33}$.

Es interesante asimismo comprender que pueden existir otros factores de riesgo identificados en la paciente potencialmente preeclamptica y que están asociados a otras entidades como la depresión y la violencia basada en género que podrían condicionar el desarrollo de preeclampsia ${ }^{34}$ y además se publicará ya una nueva publicación ACOG sobre preeclampsia en enero del $2019^{35}$.

En conclusión, el nomograma de predicción de riesgo de preeclampsia propuesto predice el desarrollo de preeclampsia con una alta sensibilidad e incluye parámetros clínicos, de laboratorio y del doppler de las arterias uterinas clasificando el riesgo en alto, intermedio y bajo. Por lo que puede usarse en la práctica clínica. 
Financiamiento: Autofinanciado

Conflicto de interés: Los autores declaran no tener algún conflicto de intereses.

\section{REFERENCIAS BIBLIOGRÁFICAS}

1. World Health Organization, UNICEF, UNFPA, The World Bank, United Nations. Trends in Maternal Mortality: 1990 to 2013. Estimates developed by WHO, UNICEF, UNFPA, The World Bank and the United Nations Population Division. Geneva: World Health Organization; 2014. Disponible en: http://apps.who.int/iris/bitstream/10665/112697/1/WHO_ RHR_14.13_eng.pdf?ua=1

2. National High Blood Pressure Education Program Working Group on High Blood Pressure in Pregnancy. Report of the National High Blood Pressure Education Program Working Group on High Blood Pressure in Pregnancy. Am J Obstet Gynecol. 2000;183(Suppl 1):S1-22.

3. Duley L. The global impact of pre-eclampsia and eclampsia. Semin Perinatol. 2009 Jun;33(3):130-7. doi: 10.1053/j. semperi.2009.02.010.

4. Ministerio de Salud. Dirección General de Epidemiología. La mortalidad materna en el Perú 2002-2012. Lima, Perú, mayo 2013.

5. Lindheimer MD, Roberts JM, Cunningham FG. Chesley's Hypertensive Disorders in Pregnancy, 3rd ed. Stamford, CT: Appleton \& Lange; 2009

6. Pacheco J, Wagner $P$, Williams $M$, Sánchez $S$. Enfermedadeshipertensivas en la gestación. En: Pacheco J. Ginecología,Obstetricia y Reproducción. 2a ${ }^{\mathrm{a}}$. Edición. Lima: REP SAC. 2007:1097-130.

7. Methodius G. Tuuli, Anthony O. Odibo .First- and SecondTrimester Screening for Preeclampsia and Intrauterine Growth Restriction. Clin Lab Med. 2010;30:727-746

8. Sibai B, Dekker G, Kupferminc M. Preeclampsia. Lancet 2005; 365:785-99

9. Van Laake LW, Van den Driesche S, Post S,et al. Endoglin has a crucial role in blood cell mediated vascular repair. Circulation. 2010; 114(4) :2288-97.

10. Gonzales CA, Alegría CR. ¿Es posible predecir la preeclampsia?. Rev. Peru. Ginecol. Obstet.; 60(4): 363-372. Disponible en: http://www.scielo.org. pe/scielo.php? script=sci_arttext\&pid=S2304$51322014000400012 \&$ Ing $=$ es.

11. Ernster VL. Nested case-control studies. Prev Med 1994; 23:587-590

12. American College of Obstetricians and Gynecologists (ACOG). Task Force on Hypertension in Pregnancy 2013. Hypertension in Pregnancy. Practice Guideline. WQ 244. Disponible en: doi: 10.1097/01.AOG.0000437382.03963.88

13. Peterson HB, Kleinbaum DG. Interpreting the literature in obstetrics and gynecology: II. Logistic regression and related issues. Obstet Gynecol.1991; 78:717-20.

14. López-Carbajal MJ et al. Factores de riesgo asociados con preeclampsia.Rev Med Inst Mex Seguro Soc 2012; 50 (5): 471-476
15. Lara-González AL, Ulloa-Galván G, Alpuche G, RomeroArauz JF. Factores de riesgo para preeclampsia. Análisis multivariado. Ginecol Obstet Mex. 2000;68(1):357-362.

16. Saftlas, A.F., Olson, D.R., Franks, A.L., Arana, Jose D., Pokras, R. Epidemiology of preeclampsia and eclampsia in the United States, 1979-1986. American Journal of Obstetrics and Gynecology. 163, (2), 460-465

17. Skjaerven R, Wilcox AJ, Lie RT. The interval betheen pregnancies and the risk of preeclampsia. $\mathrm{N}$ Engl $\mathrm{J}$ Med. 2002;346(3):33-8

18. Conde-Agudelo A, Belizán JM. Risk factors for preeclampsia in a large cohort of Latin American and Caribbean women. BJOG. 2000; 107(3):75-83.

19. Rosell-Juarte E, Brown-Bonora R, Hernández AP. Factores de riesgo de la enfermedad hipertensiva del embarazo. Arch Médico de Camagüey 2006;10(5):1-9

20. Papageorghiou AT, Yu CKH, Bindra R, Pandis G, Nicolaides $\mathrm{KN}$. Multicentre screening for pre-eclampsia and fetal growth restriction by transvaginal uterine artery Doppler at 23 weeks of gestation. Ultrasound Obstet Gynecol. 2001;18(3):441-9.

21. Patrick Emonts y col.Prediction of Maternal Predisposition to Preeclampsia. Hypertension in Pregnancy2008;27(1):237-245,

22. Pilalis A, Souza P, Antsaklis P, Daskalakis G, Papantoniou $\mathrm{N}$, Mesogitis S, Antsaklis A. Screening for preeclampsia and fetal growth restriction by uterine artery Doppler and PAPP-A at 11-14 weeks' gestation. Ultrasound Obstet Gynecol. 2007; 29(1): 135-140

23. Irfan KA, Fathima SM, Iqbal Y,Naseeruddin M. Role of Uterine Artery Doppler in 11-14 Weeks Scan as a Predictor of Preeclampsia. Ann Int Med Den Res. 2018; 4(1): 8-11.

24. Guibovich Mesinas A, Fang Marino A. Ultrasonografia doppler de arterias uterinas entre las 11 a 14 semanas de edad gestacional, como predictor de preeclampsia.Rev Horiz Med.2012;12(2):1-2

25. Huertas E, et al. Valor predictivo del índice de pulsatilidad promedio de las arterias uterinas en la predicción de preeclampsia en las gestantes entre 11 y 14 semanas, Instituto Nacional Materno Perinatal.Rev. Peru. Epidemiol. Abril 2012.16(1):1-3

26. Morgan-Ortiz F y col. Factores de riesgo asociados con preeclampsia: estudio de casos y controles. Ginecol Obstet Mex .2010;78(3):153-159

27. Demidenko E. Sample size determination for logistic regression revisited. Stat Med. 2007; 26(18):3385-97.

28. R K Morris R D Riley M Doug J Deeks.Diagnostic accuracy of spot urinary protein and albumin to creatinine ratios for detection of significant proteinuria or adverse pregnancy outcome in patients with suspected pre-eclampsia: systematic review and meta-analysis.BMJ 2012;345:e4342 doi: 10.1136/bmj.e4342.

29. Yu CK, Smith GC, Papageorghiou AT, Cacho AM, Nicolaides $\mathrm{KH}$. An integrated model for the prediction of pre-eclampsia using maternal factors and uterine artery Doppler velocimetry in unselected low-risk women. Am J Obstet Gynecol. 2006;195(1):330.

30. Kypros H. Nicolaides. Turning the Pyramid of Prenatal Care. Fetal Diagn Ther 2011;29(5):183-196 
31. Rolnik DL, Wright D, Poon LCY, Syngelaki A, O'Gorman $\mathrm{N}$, de Paco Matallana et al. ASPRE trial: performance of screening for preterm pre-eclampsia. Ultrasound Obstet Gynecol. 2017t;50(4):492-495.

32. P. Xiao, Yin YX, Gao YF, Lau S, Shen F, Zhao M, Chen Q. The increased maternal serum levels of IL-6 are associated with the severity and onset of preeclampsia. Cytokine. 2012;60(3):856-60. doi: 10.1016/jcyto.2012.07.039.

33. K.A. Eastwood, C. Patterson, A.J. Hunter, D.R. McCance, I.S. Young, V.A. Holmes Evaluation of the predictive value of placental vascularisation indices derived from 3-Dimensional power Doppler whole placental volume scanning for prediction of preeclampsia: A systematic review and meta-analysisPlacenta (2017), doi: 10.1016/j. placenta.2017.01.005.
34. Qiu C, Sanchez SE, Lam N, Garcia P, Williams MA. Associations of depression and depressive symptoms with preeclampsia: results from a Peruvian case-control study. BMC Womens Health. 2007;7:15.

35. American College of Obstetricians and Gynecologists (ACOG). Hypertension in Pregnancy 2019. In press Hypertension in Pregnancy. Practice Bulletin.2019;202 y 203.

\section{Correspondencia:}

Dirección: Alameda Eugenio Yacolevf Edificio 120, Dpto: 204 La Torres de San Borja

Email: cgonzales@iemp.gob.pe 